\title{
ON THE INTERPRETATION OF THE REDSHIFT IN A STATIC GRAVITATIONAL FIELD
}

\author{
L.B. OKUN and K.G. SELIVANOV \\ ITEP, Moscow, 117218, Russia \\ e-mail: okun@heron.itep.ru, selivano@heron.itep.ru \\ and \\ V.L. TELEGDI \\ EP Division, CERN, CH - 1211 Geneva 23 \\ e-mail: valentine.telegdi@cern.ch
}

\begin{abstract}
The classical phenomenon of the redshift of light in a static gravitational potential, usually called the gravitational redshift, is described in the literature essentially in two ways: on the one hand the phenomenon is explained through the behaviour of clocks which run the faster the higher they are located in the potential, whereas the energy and frequency of the propagating photon do not change with height. The light thus appears to be redshifted relative to the frequency of the clock. On the other hand the phenomenon is alternatively discussed (even in some authoritative texts) in terms of an energy loss of a photon as it overcomes the gravitational attraction of the massive body. This second approach operates with notions such as the "gravitational mass" or the "potential energy" of a photon and we assert that it is misleading. We do not claim to present any original ideas or to give a comprehensive review of the subject, our goal being essentially a pedagogical one.
\end{abstract}




\section{Introduction}

There are two kinds of photon redshift known in the literature, a gravitational and a cosmological one. Though in the General Relativity framework the two shifts can be described very similarly, in the literature they are usually discussed separately. The cosmological redshift is that of light from distant galaxies which recede. It is generally referred to as the Hubble redshift. For the farthest observed galaxies it is quite large, $\Delta \lambda / \lambda \approx 5$. The gravitational redshift arises when light moves away from a static massive object, e.g. the earth or the sun. Its observed magnitudes are generally small. This paper is devoted exclusively to this type of redshift.

The gravitational redshift is a classical effect of Einstein's General Relativity (GR), one predicted by him [1] well before that theory was created [2] (for the historical background, see e.g., [3]). Phenomenologically one can simply affirm that the frequency of light emitted by two identical atoms is smaller for the atom which sits deeper in the gravitational potential. A number of ingenious experiments have been performed [4] tations of this effect. They are discussed in a number of excellent reviews [10] whose main goal is to contrast the predictions of GR with those of various non-standard theories of gravity. Explanations of the gravitational redshift per se within the standard framework are however not critically discussed in these reviews.

Most treatises on GR [11], [12] follow the definitive reasoning of Einstein [2] according to which the gravitational redshift is explained in terms of universal property of standard clocks (atoms, nuclei). The proper time interval between events of emission of two photons as measured by the standard clock at the point of emission is different from the proper time interval between events of absorption of those photons as measured by identical standard clock at the point of absorption (in this way it was first formulated in [13]).

In the static gravitational potential the picture simplifies because there is a distinguished time - the one on which metric is independent. This time can be chosen as a universal (world) one. Under this choice the energy difference between two atomic levels increases with the distance of the atom from the earth while the energy of the propagating photon does not change. (In what follows we speak of the earth, but it could be any other massive body.) Thus 
what is called the redshift of the photon is actually a blueshift of the atom. As for the proper times at different points, they are related to the universal time via a multiplier which depends on gravitational potential and hence has different values at different points (see section 4).

Actually, most modern textbooks and monographs [14 derive the redshift by using sophisticated general relativity calculations, e.g. using orthonormal bases (a sequence of proper reference frames) to define photon energy and parallel transporting the photon's 4-momentum along its world-line. Sometimes this description is loosely phrased as a degradation of the photon's energy as it climbs out of gravitational potential well. (Some other classical textbooks also use this loose phrasing [15].) However, the non-experts should be warned that the mathematics underlying this description is radically different from the heuristic (and wrong) arguments presented in many elementary texts, e.g. [16]. These authors claim to deduce the "work against gravity" viewpoint by pretending that the photon is like a normal, low-velocity, massive particle and thus has a "photon mass" and "photon potential energy". Such derivations are incorrect and should be avoided. They are in fact avoided in exceptional popular texts, e.g. [17].

\section{Experiments}

The first laboratory measurement of the gravitational redshift was performed at Harvard in 1960 by Robert Pound and Glenn Rebka [4], [5] (with 10\% accuracy) and in 1964 by Pound and Snider [6] (with 1\% accuracy). The photons moved in a 22.5-meter tall tower. The source and the absorber of the photons ( $\gamma$-rays of $14.4 \mathrm{keV}$ energy) were ${ }^{57} \mathrm{Fe}$ nuclei. The experiment exploited the Mössbauer effect which makes the photon lines in a crystal extremely monochromatic. The redshift was compensated through the Doppler effect, i.e., by slowly moving the absorber and thereby restoring the resonant absorption. The shift measured in this way [5] was $\Delta \omega / \omega \approx 10^{-15}$.

As to the interpretation of the result, there is some ambiguity in the papers by Pound et al. Although they mention the clock interpretation by referring to Einstein's original papers, the absolute reddening of the photon is also implied as can be seen from the title of Pound's talk in Moscow [5] "On the weight of photons". From the title of the paper by Pound and Snider [6], "Effect of gravity on nuclear resonance" one might infer that they did not want to commit 
themselves to any interpretation.

By contrast, the majority of the reviews of gravitational experiments [10] quote the Harvard result as a test of the behaviour of clocks. In fact the result must be interpreted as a relative shift of the photon frequency with respect to the nuclear one since the experiment does not measure these frequencies independently.

An experiment measuring the relative shift of a photon (radio-wave) frequency with respect to an atomic one was also performed with a rocket flying up to 10,000 $\mathrm{km}$ above the earth and landing in the ocean [7].

Alongside the experiments [3]-[7] special measurements of the dependence of the atomic clock rate on the altitude were done directly by using airplanes [8], [9] (see also reviews [10]). In these experiments a clock which had spent many hours at high altitude was brought back to the laboratory and compared with its "earthly twin". The latter, once corrected for various background effects, lagged behind by $\Delta T=\left(g h / c^{2}\right) T$, where $T$ is the duration of the flight at height $h, g$ the gravitational acceleration, and $c$ the speed of light.

One of these background effects is the famous "twin paradox" of Special Relativity, which stems from the fact that moving clocks run slower than clocks at rest. It is easy to derive a general formula which includes both the gravitational potential $\phi$ and the velocity $u$ (see, e.g., the book by C. Möller [11):

$$
d \tau=d t\left[1+2 \phi / c^{2}-u^{2} / c^{2}\right]^{1 / 2}
$$

where $\tau$ is the proper "physical" time of the clock, while $t$ is the so-called world time, which can be introduced in the case of a static potential and which is sometimes called laboratory time.

In his lectures on gravity [12], Richard Feynman stresses the differences between the effects due to $u$ and to $\phi$. He concludes that the centre of the earth must be by a day or two younger than its surface. 


\section{Theory before General Relativity: "elevators"}

Since most of the conclusive experiments on the gravitational redshift were earthbound, we shall throughout use that frame in which the earth is at rest (neglecting its rotation).

As is well known, a potential is defined up to a constant. When considering the gravitational potential $\phi(r)$ at an arbitrary distance $r$ from the earth's centre, it is convenient to set $\phi(\infty)=0$; then $\phi$ is negative everywhere.

Near the earth's surface (at $h=r-R \ll R$ ) it is legitimate to approximate $\phi$ linearly:

$$
\delta \phi(h)=\phi(R+h)-\phi(R)=g h,
$$

where $g$ is the usual gravitational acceleration. Note that $\delta \phi(h)$ is positive for $h \neq 0$. We shall discuss the redshift only to the first order in the parameter $g h / c^{2}$.

(The linear approximation Eq. (2) is valid for the description of experiments [8, 9]. It is obvious, however, that for the high-flying rockets [7] $\left(h \approx 10^{4} \mathrm{~km}\right)$ it is not adequate and the newtonian potential should be used, but this is not essential for the dilemma "clocks versus photons" which is the subject of this paper.)

Einstein's first papers [1] on the gravitational redshift contain many of the basic ideas on the subject which were incorporated (sometimes without proper critical analysis) into numerous subsequent texts. He considered the Doppler effect in the freely falling frame and found the increase of the frequency of an atom (clock) with increasing height (potential). The cornerstone of his considerations was the local equivalence between the behaviour of a physical system in a gravitational field and in a properly accelerated reference frame.

For the potential (2) it is particularly convenient to appeal to Einstein's freely falling reference frame ("elevator"). In such an elevator an observer cannot detect any manifestation of gravity by any strictly local experiment (equivalence principle). Operationally, "strictly local" means that the device used is sufficiently small not to be sensitive to curvature effects.

Consider from such an elevator falling with the acceleration $g$ a photon of frequency $\omega$ which is emitted upwards by an atom at rest on the surface of the earth and which is expected to be absorbed by an identical atom fixed at height $h$. The frequency of light is not affected by any 
gravitational field in a freely falling elevator: it keeps the frequency with which it was emitted. Assume that at the moment of emission $(t=0)$ the elevator had zero velocity. At the time $t=h / c$, when the photon reaches the absorbing atom, the latter will have velocity $v=g h / c$ directed upwards in the elevator frame. As a result the frequency of the photon, as seen by the absorbing atom, will be shifted by the linear Doppler effect by $v / c=g h / c^{2}$ towards the red, that is

$$
\frac{\Delta \omega}{\omega}=-\frac{g h}{c^{2}}
$$

(Minute corrections of higher order in $g h / c^{2}$ to the "elevator formulas" are lucidly discussed on the basis of a metric approach in ref. [18].) Consider now another situation, when the upper atom (absorber) moves in the laboratory frame with a velocity $v=g h / c$ downwards. Then in the elevator frame it will have zero velocity at the moment of absorption and hence it will be able to absorb the photon resonantly in complete agreement with experiments [4, 5]. Obviously, in the elevator frame there is no room for the interpretation of the redshift in terms of a photon losing its energy as it climbs out of the gravitational well.

\section{Theory in the framework of General Relativity: metric}

Up to now we used only Special Relativity and newtonian gravity. As is well known, a consistent relativistic description of classical gravity is given in the framework of GR with its curved space-time metric. One introduces a metric tensor, $g_{i k}(x), i, k=0,1,2,3$, which is, in general, coordinate dependent and transforms by definition under a change of coordinates in such a way that the interval $d s$ between two events with coordinates $x^{i}$ and $x^{i}+d x^{i}$,

$$
d s^{2}=g_{i k}(x) d x^{i} d x^{k}
$$

is invariant. Setting $d x^{1}=d x^{2}=d x^{3}=0$, one obtains the relation between the proper time interval $d \tau=d s / c$ and the world time interval $d t=d x^{0} / c$ for an observer at rest

$$
d \tau=\sqrt{g_{00}} d t
$$

For a static case, Eq. (5) integrates to

$$
\tau=\sqrt{g_{00}} t
$$


where $g_{00}$ is a function of $\boldsymbol{x}$ in the general case while in the case of Eq.(2) it is a function of $x^{3}=z=h$.

The time $\tau$ is displayed by a standard clock and can also be viewed on as a time coordinate in the so-called comoving locally inertial frame, i.e. such locally inertial frame which at a given instant has zero velocity with respect to the laboratory frame. If one has a set of standard clocks at different points, then their proper times $\tau$ are differently related to the world (laboratory) time $t$, due to $\boldsymbol{x}$-dependence of $g_{00}$ (see Eq. (6)). This explains the airplane experiments [8], [9]. Let us recall that sometimes the world time is called laboratory time. The former term reflects the fact that it is the same for the whole world, the latter signifies that it can be set with standard clocks in the laboratory. Many authors refer to $t$ as the coordinate time.

A weak gravitational field can be described by a gravitational potential $\phi$, and $g_{00}$ is related to the gravitational potential:

$$
g_{00}=1+2 \phi / c^{2}
$$

We shall explain the meaning of this relation a bit later (see Eqs. (8)-(10)). According to Eqs. (6), (7) a clock runs the slower in the laboratory the deeper it sits in the gravitational potential.

Analogous to Eq. (5) is a relation between the rest energy $E_{0}$ of a body in the laboratory frame and in the comoving locally inertial frame,

$$
E_{0}^{l a b}=E_{0}^{l o c} \sqrt{g_{00}}
$$

(notice that $E_{0}^{l a b} d t=E_{0}^{l o c} d \tau$; this is because the energy $E$ is the zero-th component of a covariant 4 -vector, while $d t$ is the zero-th component of a contravariant 4 -vector).

The rest energy in the locally inertial frame is the same as in the special relativity (see e.g. [19] and the book by E. Taylor and J.A. Wheeler [16], p.246).

$$
E_{0}^{l o c}=m c^{2}
$$

while the rest energy in the laboratory system $E_{0}^{l a b}$ includes also the potential energy of the body in the gravitational field. This is in accordance with the main principle of the general relativity: all effects of gravity arise only via the metric tensor. Eq. (7) for the $g_{00}$ component of the metric tensor in a weak gravitational field can be considered as a consequence of Eqs. 
(8), (9) and of the relation

$$
E_{0}^{l a b}=m c^{2}+m \phi
$$

which generalizes the notion of the rest energy of a free particle to that of a particle of mass $m$ in a gravitational weak potential.

Now we are in a position to explain the redshift in the laboratory frame. According to Eq. (8) or Eq. (10) the energy difference $\varepsilon_{l a b}$ of atomic or nuclear levels in that frame depends on the location of the atom. The deeper atom sits in the gravitational potential the smaller is $\varepsilon_{l a b}$. For an absorber atom which is located at height $h$ above an identical atom which emits the photon, the relative change in the energy difference is $g h / c^{2}$,

$$
\frac{\Delta \varepsilon^{l a b}}{\varepsilon^{l a b}}=\frac{g h}{c^{2}} \text {. }
$$

(We use in Eq. (11) the linear approximation of Eq. (2).) One can say that the energy levels of the absorber atoms are shifted towards the blue in the laboratory frame. Eq. (11) is, of course, nothing but a way to describe the difference in the rates of atomic clocks located at a height $h$ one above the other. On the other hand, the energy (frequency) of the photon is conserved as it propagates in a static gravitational field. This can, for example, be seen from the wave equation of electromagnetic field in the presence of a static gravitational potential or from the equations of motion of a massless (or massive) particle in a static metric. Clearly, in the laboratory system there is no room for the interpretation in which the photon loses its energy when working against the gravitational field.

Finally, one can discuss the experiment using a sequence of locally inertial frames which are comoving with the laboratory clocks (atoms) at the instants when the photon passes them. As we explained above, in such systems the standard clocks run with the same rates, the rest energy of the atom is equal to its mass, times $c^{2}$, Eq. (9), and the energy levels of the atom are the same as at infinity. On the other hand, the energy of the photon in the laboratory frame $E_{\gamma}^{l a b}=\hbar \omega^{l a b}$ and in the comoving locally inertial frame $E_{\gamma}^{l o c}$ are related as

$$
E_{\gamma}^{l a b}=E_{\gamma}^{l o c} \sqrt{g_{00}}
$$

Eq.(12) follows from Eq.(8) by noticing that the photon can be absorbed by a massive body and by considering the increase of the rest energy of that body. Thus, since $E_{\gamma}^{\text {lab }}$ is conserved, 
$E_{\gamma}^{l o c}$ decreases with height:

$$
\frac{\omega^{l o c}(h)-\omega^{l o c}(0)}{\omega^{l o c}(0)}=\frac{E_{\gamma}^{l o c}(h)-E_{\gamma}^{l o c}(0)}{E_{\gamma}^{l o c}(0)}=-\frac{g h}{c^{2}}
$$

and this is the observed redshift of the photon. But $E^{l o c}$ decreases not because the photon works against the gravitational field. The gravitational field is absent in any locally inertial

frame. $E_{\gamma}^{l o c}$ changes because one passes from one of the locally inertial frames to the other: the one comoving with the laboratory at the moment of emission, the other - at the moment of absorption.

\section{$5 \quad$ Pseudoderivation and misinterpretation of gravitational redshift}

The simplest (albeit wrong) explanation of the redshift is based on ascribing to the photon both an inertial and a gravitational " mass" $m_{\gamma}=E_{\gamma} / c^{2}$. Thereby a photon is attracted to the earth with a force $g m_{\gamma}$, while the fractional decrease of its energy (frequency) at height $h$ is

$$
\frac{\Delta E_{\gamma}}{E_{\gamma}}=\frac{\Delta \omega}{\omega}=-g m_{\gamma} h / m_{\gamma} c^{2}=-g h / c^{2} .
$$

Note that (up to a sign) this is exactly the formula for the blueshift of an atomic level. That should not surprise. The atom and the photon are treated here on the same footing, i.e. both non-relativistically! This is of course inappropriate for the photon. If the explanation in terms of gravitational attraction of the photon to the earth were also correct, then one would be forced to expect a doubling of the redshift (the sum of the effects on the clock and on the photon) in the Pound-type experiments.

Some readers may invoke Einstein's authority to contradict what was said above. In his 1911 paper [1], he advanced the idea that energy is not only a source of inertia, but also a source of gravity. Loosely speaking, he used the heuristic argument "whenever there is mass, there is also energy and vice versa". As he realized later, this "vice versa" was not as correct as the direct statement (a photon has energy while its mass is zero). By applying this energy-mass argument, he calculated the energy loss of a photon moving upwards in the potential of the 
earth as discussed above. With the same heuristic principle he also derived an expression for the deflection of light by the sun which however underestimated the deflection angle by a factor of 2. Subsequently, in the framework of GR, Einstein recovered this factor [2]. The correct formula was confirmed by observation.

\section{The wavelength measurement}

Up to now we have discussed the gravitational redshift in terms of the photon frequency and clocks. Let us now consider the same phenomenon in terms of the photon wavelength and gratings. To do this we consider two identical gratings at different heights, inclined with respect to the $z$-axis along which the light propagates between them. We do not go into the details of this gedanken experiment. The $z$-projections of grating spacing is used only as a standard of length. The lower grating serves as monochromator, i.e. as the light source. The wavelength of the photon $\lambda^{l a b}(z)$ corresponds to its frequency, while the spacings of the grating in vertical $(z)$ direction $l^{l a b}(z)$ correspond to the rates of the clocks.

For the sake of simplicity, one may consider a very small incidence angle on the gratings, i.e. the grazing incidence of the light. In that case, the vertical projection of the spacing is practically the spacing itself. (Recall that, for the grazing incidence, the spacing $l^{l a b}$ must be of the same order as the wavelength $\lambda^{l a b}$.)

While the photon energy $E^{l a b}$ is conserved in a static gravitational field the photon momentum $p^{l a b}$ is not. The relation between these quantities is given by the condition that the photon remains massless which in a gravitational field reads

$$
g^{i j} p_{i} p_{j}=0
$$

where the $g^{i j}, i, j=0, \ldots, 3$ are the contravariant components of the metric tensor, $p_{j}$ are the

components of the 4-momentum, $p_{0}=E^{l a b}, p_{3}=p^{l a b}=2 \pi \hbar / \lambda^{l a b}(z)$ (for the photon moving along the $z$-axis). For the cases we are discussing the metric $g^{i j}$ can be taken in diagonal form, in particular $g^{33}=1 / g_{33}$.

From Eq. (15) one readily finds how $\lambda^{l a b}(z)$ changes with height:

$$
\lambda_{l a b}(z)=\sqrt{g^{33}(z) / g^{33}(0)} \sqrt{g^{00}(0) / g^{00}(z)} \lambda_{l a b}(0)
$$


On the other hand, the grating spacing in the $z$-direction, $l^{l a b}(z)$, also changes with height. This is just the standard change of scale in the gravitational field, explained e.g. in the book by L. Landau and E. Lifshitz, $\S 84$, 11]:

$$
l^{l a b}(z)=\sqrt{-g^{33}(z)} l^{0}
$$

where $l^{0}$ is the "proper spacing" in $z$-direction, counterpart of the proper period of the standard clock. Thus the spacing $l^{l a b}(z)$ depends on $z$ as follows

$$
l^{l a b}(z)=\sqrt{g^{33}(z) / g^{33}(0)} l^{l a b}(0)
$$

Finally, in the wavelength analogue of the Pound et al. experiment with $z=h$ one would measure the double ratio $(\lambda(h) / l(h)) /(\lambda(0) / l(0))$. The result can be presented in the form

$$
\Delta \lambda^{l a b} / \lambda^{l a b}-\Delta l^{l a b} / l^{l a b}=\sqrt{g^{00}(0) / g^{00}(h)}=g h / c^{2}
$$

where $\Delta \lambda^{l a b} / \lambda^{l a b}=\left[\lambda^{l a b}(h)-\lambda^{l a b}(0)\right] / \lambda^{l a b}(0)$ and analogously for $\Delta l^{l a b} / l^{l a b}$. Notice that $g^{33}$ drops out from the result. This should be so because there is a freedom in the choice of $z$-scale, and the observed quantities cannot depend on this choice. The Eq. (19) is analogous to the one describing the Pound et al. experiments:

$$
\Delta \omega / \omega-\Delta \epsilon / \epsilon=\sqrt{g_{00}(0) / g_{00}(h)}=-g h / c^{2}
$$

where $\omega$ is the frequency of the photon, $\epsilon / \hbar$ is the frequency of the clock (see Eq. (11). A word of explanation should be added about Eq. (20). In the laboratory frame the first term in the left hand side is equal to zero,

$$
\Delta \omega^{l a b} / \omega^{l a b}=0
$$

as discussed in section 3, thus only the second term given by Eq. (11) contributes.

We would however like to stress an important difference as compared to the case of frequency. There one can independently measure the difference in rates of the upper and lower clocks, $\Delta \epsilon^{l a b} / \epsilon^{l a b}$, (analogue of the second term in the left hand side of Eq. (19)) and that was done in the airplane experiments. Here the change of the scale, $\Delta l^{l a b} / l^{l a b}$, cannot be measured independently. This important difference comes from the fact that the metric is static while it is $z$-dependent. 
One has to realize that such a laboratory experiment with gratings cannot be performed at the present state of the art in experimental physics (recall the importance of Mössbauer effect in the experiments of Pound et al.). However for the measurement of a large value of the redshift, e.g. that of sodium spectral line from the sun, it is feasible. Such a grating experiment was performed by J.W. Brault in 1962 and was described in $§ 38.5$ in the monograph by C. Misner, K. Thorne and J.A. Wheeler [14]. In this experiment the wavelength of the emitted light was fixed not by the lower grating, but by the atom on the sun surface.

\section{Conclusions}

The present article contains little original material; it is primarily pedagogical. The gravitational redshift being, both theoretically and experimentally, one of the cornerstones of General Relativity, it is very important that it always be taught in a simple but nevertheless correct way. That way centers on the universal modification of the rate of a clock exposed to a gravitational potential. An alternative explanation in terms of a (presumed) gravitational mass of a light pulse - and its (presumed) potential energy - is incorrect and misleading. We exhibit its fallacy, and schematically discuss redshift experiments in the framework of the correct approach. We want to stress those experiments in which an atomic clock was flown to, and kept at, high altitude and subsequently compared with its twin that never left the ground. The traveller clock was found to run ahead of its earthbound twin. The blueshift of clocks with height has thus been exhibited as an absolute phenomenon. One sees once over again that the explanation of the gravitational redshift in terms of a naive "attraction of the photon by the earth" is wrong.

\section{Acknowledgements}

We would like to thank V.V. Okorokov, who asked the question on compatibility in the framework of General Relativity of experiments by Pound et al., and the airplane experiments. We also thank S.I. Blinnikov, A.D. Dolgov, A.Yu. Morozov, N. Straumann, K. Thorne and G. Veneziano for very interesting discussions. We would like to express our gratitude especially to

E.L. Schucking for his help in substantially improving our bibliography and for his insistence on 
a unified invariant approach to both gravitational and cosmological redshifts based on Killing vectors. We did however not follow his advice wanting to focus the general reader's attention on the fallacy of the wide-spread naive interpretation of the gravitational redshift. Last but not least we want to thank J.A. Wheeler for his encouragement. One of us (L.O.) would like to thank the Theoretical Physics Division of CERN, where part of this work was done, for their hospitality.

\section{References}

[1] A. Einstein, "Über das Relativitätsprinzip und die aus demselben gezogenen Folgerungen," Jahrb. d. Radioaktivität u. Elektronik 4, 411-462 (1907); "Über den Einfluss der Schwerkraft auf die Ausbreitung des Lichtes," Ann. Phys. 35, 898-908 (1911).

[2] A. Einstein, "Die Grundlage der allgemeinen Relativitätstheorie," Ann. Phys. 49, 769-822 (1916) §22; The Meaning of Relativity (Princeton University Press, New York, 1921), Eq. (106).

[3] A. Pais, 'Subtle is the Lord ...', The Science and the Life of Albert Einstein (Oxford University Press, Oxford, 1982), Chapter 9.

[4] R.V. Pound and G.A. Rebka, "Apparent weight of photons," Phys. Rev. Lett. 4, 337-341 (1960); "Variation with temperature of the energy of recoil-free gamma rays from solids," Phys. Rev. Lett. 4, 274-275 (1960); "Gravitational red-shift in nuclear resonance," Phys. Rev. Lett. 3, 439-441 (1959).

[5] R. Pound, Uspekhi Fiz. Nauk 72, 673-683 (1960); "On the weight of photons," Sov. Phys. Uspekhi 3, 875-883 (1961) (English translation).

[6] R.V. Pound and J.L. Snider, "Effect of Gravity on Gamma Radiation," Phys. Rev. B140, 788-803 (1965); "Effect of gravity on nuclear resonance," Phys. Lett. 13, 539-540 (1964).

[7] R. Vessot and M. Levine, "A test of the equivalence principle using a space-borne clock," Gen. Rel. Grav. 10, 181-204 (1979). 
[8] J. Haefele and R. Keating, "Around the world atomic clocks: predicted relativistic gains," Science 177, 166-167 (1972); "Around the world relativistic clocks: observed relativistic time gains," ibid., 168-170.

[9] C. Alley et al., in Experimental Gravitation, Proceedings of the Conference at Pavia (Sept. 1976), ed. B. Bertotti (Academic Press, New York, NY, 1977).

[10] C. Will, Theory and Experiment in Gravitational Physics (Cambridge University Press, Cambridge, 1981), Section 2.4; "The confrontation between general relativity and experiment: a 1992 upgrade," Int. J. Mod. Phys. D1, 13-68 (1992); preprint WUGRAV-95-5, gr-qc/9504017 (1995);

I. Shapiro, Gravitazione Quanti e Relatività. Astrofisica e Cosmologia (Giunti Barbera, Florence, 1979), Section 3;

N. Ashby and J. Spilker, The Global Positioning System: Theory and Applications 1, (American Institute of Aeronautics and Astronautics Inc., Washington, D.C., 1995), Chapter 18;

J. Taylor, Astronomical and Space Experiments to Test Relativity, in General Relativity and Gravitation (Cambridge University Press, Cambridge, 1987), p. 214.

[11] W. Pauli, Theory of Relativity (Pergamon Press, Oxford, 1967), Section 53.

C. Möller, The Theory of Relativity (Clarendon Press, Oxford, 1960), §§92, 93.

L. Landau and E. Lifshitz, The Classical Theory of Fields (Pergamon Press, Oxford, 1962), $\S 89$.

S. Weinberg, Gravitation and Cosmology (John Wiley \& Sons, New York, NY, 1972), pp. 79-85;

P. Schneider, J. Ehlers, E.E. Falco, Gravitational Lenses (Springer-Verlag, Berlin, 1996), pp. 93-97

N. Straumann, General Relativity and Relativistic Astrophysics (Springer-Verlag, Berlin, 1984), pp. 96-99

I. Ciufolini and J.A. Wheeler, Gravitation and Inertia (Princeton University Press, Princeton, 1995), pp. 97-108.

[12] R.P. Feynman, Lectures on Gravitation, ed. Brian Hatfield (Addison-Wesley Publ. Co., Reading, MA, 1995), Section 5.2. 
[13] H. Weyl, Raum, Zeit, Materie (Verlag von Julius Springer, Berlin, 1923), 5te Auflage, p.322; see also the 7th edition republished by J. Ehlers (Springer-Verlag, Berlin, 1988).

[14] C. Misner, K. Thorne and J. A. Wheeler, Gravitation (Freeman and Co., San Francisco, 1973), Sections 7.2-7.5, 25.3, 25.4, 38.5;

R. W. Wald, General Relativity (University of Chicago Press, Chicago and London, 1984) p.137;

H. Stephani, General Relativity (Cambridge University Press, Cambridge, 1996), second edition;

I. R. Kenyon, General Relativity (Oxford University Press, Oxford, 1996), pp. 15-21.

[15] Max Born, Einstein's Theory of Relativity (Dover Publications, Inc., New York, 1962), Chapter VII, §11;

D. W. Sciama, The Physical Foundations of General Relativity (Doubleday and Company, Inc., Garden City, NY, 1969), Chapter 5;

Ya. B. Zeldovich and I.D. Novikov, Relativistic Astrophysics. Vol. 1. Stars and Relativity (University of Chicago Press, Chicago and London, 1971), pp. 89-93.

[16] The New Encyclopedia Britannica 20, p.174 (1994), 15th Edition;

J. A. Wheeler, A Journey into Gravity and Spacetime (Scientific American Library, New York, NY, 1990), pp. 48-53, 166-168;

E. Taylor and J.A. Wheeler, Spacetime Physics (Freeman and Co., New York, 1992), 3rd edition, pp. 258, 272;

D. Layzer, Constructing the Universe (Scientific American Library, New York, 1984), Chapter 6, pp. 210-212.

[17] J. Schwinger, Einstein's Legacy (Scientific American Library, New York, NY, 1986), pp. $141-153$.

[18] E.A. Desloge, "Nonequivalence of a uniformly accelerating reference frame and a frame at rest in a uniform gravitational field," Am. J. Phys. 57, 1121-1125 (1989); "The gravitational red shift in a uniform field," Am. J. Phys. 58, 856-858 (1990).

[19] L. B. Okun, "The Concept of Mass," Physics Today, pp. 31-36 (June 1989); "Putting to Rest Mass Misconceptions," Physics Today, pp. 13,15,115,117 (May 1990); Usp. Fiz. Nauk 
158, 511-530 (1989); "The concept of mass (mass, energy, relativity)," Sov. Phys. Usp. 32, 629-638 (1989); "Note on the meaning and terminology of Special Relativity," Eur. J. Phys. 15, 403-406 (1998). 